\title{
A Comparison of Rey-Osterrieth Complex Figure Test Scores: With/Without Mouthpiece and with/without Noise
}

\author{
Masako Notoya ${ }^{1 *}$, Katsumi Inoue ${ }^{2}$, Ai Hirabayashi' ${ }^{3}$, Kana Sakamoto $^{4}$, Chihiro Sasaguchi ${ }^{5}$, \\ Minoru Toyama ${ }^{1,2}$ \\ ${ }^{1}$ Department of Speech and Hearing Sciences and Disorders, Kyoto Gakuen University, Kyoto, Japan \\ ${ }^{2}$ Graduate School of Medical Sciences, College of Medical, Pharmaceutical and Health Sciences, Kanazawa University, Ishikawa, Japan \\ ${ }^{3}$ Asanogawa Hospital, Kanazawa, Japan \\ ${ }^{4}$ Saiseikai Hospital, Kanazawa, Japan \\ ${ }^{5}$ Hikone Chuo Hospital, Shiga, Japan \\ Email: ^notoya@kyotogakuen.ac.jp, tsubura1@staff.kanazawa-u.ac.jp, 93kxx27xxa12.gm@gmail.com, sakana1428@yahoo.co.jp, \\ os-ax5.one@ezweb.ne.jp, toyama@kyotogakuen.ac.jp
}

How to cite this paper: Notoya, M., Inoue, K., Hirabayashi, A., Sakamoto, K., Sasaguchi, C. and Toyama, M. (2017) A Comparison of Rey-Osterrieth Complex Figure Test Scores: With/Without Mouthpiece and with/without Noise. World Journal of Neuroscience, 7 , 282-292.

https://doi.org/10.4236/wjns.2017.73024

Received: April 24, 2017

Accepted: July 17, 2017

Published: July 20, 2017

Copyright $\odot 2017$ by authors and Scientific Research Publishing Inc. This work is licensed under the Creative Commons Attribution International License (CC BY 4.0).

http://creativecommons.org/licenses/by/4.0/

\section{(c) (i) Open Access}

\begin{abstract}
In the present study, the ROCF test was initially conducted involving 30 healthy young individuals, in a quiet environment as Experiment 1 to examine variations in the score among different methods to memorize the figure. In such an environment, no significant differences were observed in the score between the copying and outer speech groups, which suggested the possibility of some of the former groups having used outer speech in a voice too low to be heard or moving their lips without vocalization, achieving the same effect as outer speech, and consequently leading to the absence of differences from the outer speech group. On the other hand, the score markedly varied between the mouthpiece and copying or outer speech groups. As lip movements were suppressed in the former case, the unconscious use of outer speech was also prevented, possibly leading to poor results. Based on these findings, it may be possible to enhance the effects of rehabilitation in a clinical setting by promoting patients' memorization using outer speech to vocalize the contents of training.
\end{abstract}

\section{Keywords}

ROCF Test, Promotion Visual Memory, Outer Speech

\section{Introduction}

The Rey-Osterrieth complex figure test (ROCFT) is one of the most commonly 
used neuropsychological tests for assessing visuospatial construction ability and visual memory [1]. Lu et al. described recognition scores of the ROCFT [2]. This test involves copying a complex geometric figure and then reproducing it from memory, either immediately or after a delay, in patients with brain injury. It is widely used in a clinical setting.

Studies using the ROCFT have revealed visual memory disturbance in individuals with schizophrenia [3]. Similarly, individuals with Alzheimer's disease (AD) and Korsakoffs syndrome have shown poorer copy and recognition on the ROCFT than controls [4]. Cuyas, Verdejo-Garcia, Fanundo, et al. attempted to clarify the association between 3, 4-methylenedioxymethamphe-tamine (MDMA) cumulative use and cognitive dysfunction [5]. They found that lifetime cumulative MDMA use was significantly associated with poorer performance on visuospatial memory by ROCFT.

In addition, Theppitak, Lai, Izumi, et al. reported that visual recognition and memory performance for elderly persons were improved by extending the encoding time and performing repeated test trails [6]. Moreover, Yamashita (2008) reported that repeated administration of the ROCFT continued significant practice effects for 12 months. In conclusion, a more careful attitude was required for repeated administration of the ROCFT and interpretation of the results [7].

A few reports have described that the effectiveness of strategies to promote immediate visual memory and delayed recall is measurable using the ROCF test. The results of ROCFT have been reported to vary depending on the memorization method or environmental conditions. For example, the effects of outer speech in a quiet environment were confirmed by Lezak (2004), with "outer speech" referring to a method of expressing one's own behavior by vocalizing it [8]. We hypothesized that the performance of visual memory is reduced by blocking outer speech.

Two experiments were conducted using the ROCF test. In Experiment 1, the figure was copied under 3 conditions prior to its reproduction: copying it as usual; using outer speech; and holding a mouthpiece in the mouth to prevent such speech. The figure was reproduced and assessed in a quiet environment 3 minutes and 3 days after copying.

In Experiment 2, the test was also conducted under the same conditions, but with noise. As the clinical rehabilitation setting tends to involve various types of noise, the necessity of examining the influence of noise on these conditions was considered.

\section{Methods of Experiment 1}

\subsection{Subjects}

Thirty (6 males and 24 females with a mean age of $21.20 \pm 1.80$ years) students belonging to the study university, Kanazawa University, were examined with their consent. All the students were high level university students. They have no mental disorders. They were divided into 3 groups: copying, outer speech, and mouthpiece groups. Each group was made up of 10 members, at the same sex ra- 
tio.

This study was conducted with the approval of the Medical Ethics Committee of Kanazawa University.

\subsection{Method 1}

The test was conducted on a face-to-face basis in a quiet, private room without noise (quiet environment) in all cases. The ROCF figure was reproduced $3 \mathrm{mi}$ nutes and 3 days after copying it under 3 conditions for memorization: copying as usual; using outer speech; and holding a mouthpiece in the mouth. The outer speech group copied the figure while vocalizing the process of copying, while the mouthpiece group copied it with a mouthpiece held in their mouths.

After copying the figure, all of the subjects were instructed to participate in a discussion to prevent them from rehearsing the reproduction of the figure. They were also instructed to reproduce it after 3 days without notification. The reproduced figure was scored by the examiner based on appropriate criteria. The maximum full score was 36 .

\subsection{Questionnaire Survey}

A questionnaire survey was conducted after the completion of the task to examine the subjects' reflection on each condition through free recall.

\subsection{Statistical Analysis}

To compare time-dependent changes in the score under each condition, a 2-way factorial analysis of variance was performed, with the condition as an unpaired factor and the time as a paired factor. This was followed by multiple comparisons using Tukey's method.

\section{Results of Experiment 1}

\subsection{Mean Scores under Each Condition}

Table 1 shows the mean scores and standard deviations of each group at $3 \mathrm{mi}$ nutes and 3 days after copying. The copying and outer speech groups showed the highest scores at the former and the latter, respectively. The mouthpiece group showed the lowest score at both time points. Furthermore, the score at the latter was lower than that at the former under all conditions. There are significant dif-

Table 1. Mean scores and standard deviations of each group at 3 minutes and 3 days after copying (without noise).

\begin{tabular}{ccc}
\hline & \multicolumn{2}{c}{ Mean (SD) } \\
\cline { 2 - 3 } & 3 minutes later & 3 days later \\
\hline Copying group & $28.95(4.21)$ & $24.55(5.1)^{*}$ \\
Outer speech group & $27.95(4.98)$ & $25.2(6.11)^{*}$ \\
Mouthpiece group & $23.55(5.44)$ & $21.2(5.41)^{*}$
\end{tabular}

The maximum full score was $36 .{ }^{*} p<0.05$. 


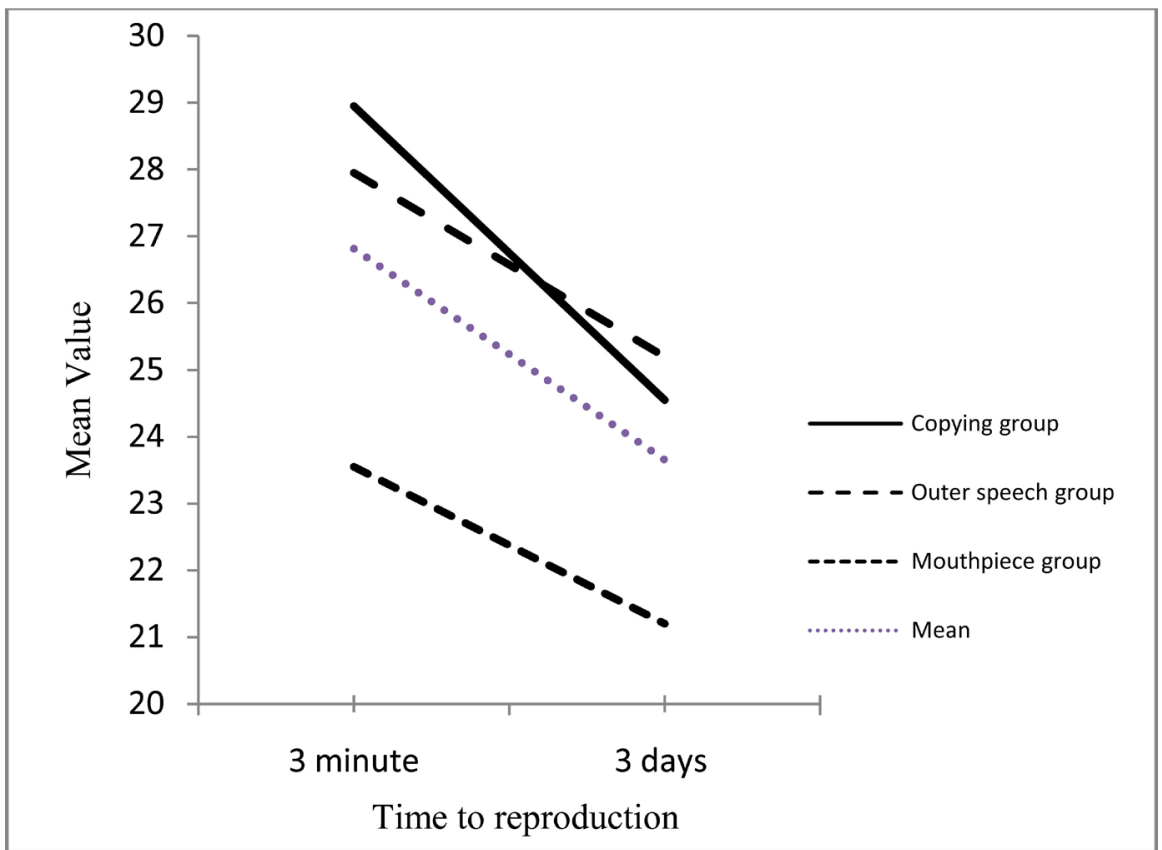

No interactions were observed under each condition. The mean differences in scores between $3 \mathrm{mi}-$ nutes and 3 days after each group were tested ${ }^{\star} p<0.05$.

Figure 1. Time-dependent changes in the score of each group (without noise).

ference between copying group and mouth piece group, between outer speech group and mouthpiece group, but not different between copying group and outer speech group.

\subsection{Time-Dependent Changes in the Score under Each Condition}

No interactions were observed under each condition. The score varied markedly between 3 minutes and 3 days after copying ( $p<0.05$, Figure 1$)$. There were also significant differences between the copying or outer speech and mouthpiece groups $(p<0.05)$, but such differences were not observed between the copying and outer speech groups.

\subsection{Questionnaire Survey}

Seven out of the 10 outer speech group members made a positive evaluation of outer speech, such as "Vocalization facilitated recall" and "The figure remained in my memory". In contrast, 5 out of the 10 mouthpiece group members made negative comments, such as "The presence of the mouthpiece distracted me" and "There was an uncomfortable feeling".

\section{Brief Discussion}

In this experiment, the ROCF test was conducted, involving 30 healthy young individuals copying the figure as follows: as usual, using outer speech, or holding a mouthpiece in the mouth. Gallagher \& Burke [9] pointed out IQ effects on the ROCFT score. But all the participants were all good level university students and no IQ effect in these results. 
The figure was reproduced and scored after 3 minutes and 3 days to examine delayed reproduction. There were no significant differences in the score between the copying and outer speech groups, but they were observed between the copying or outer speech and mouthpiece groups. The results are discussed in detail in the following paragraphs.

\section{Comparison between Copying and Outer Speech}

In Experiment 1, the score did not markedly vary between the copying and outer speech groups. This is similar to the results of a previous study by Tsutsui, et al. [10] in which the absence of differences between inner and outer speech groups was regarded to have resulted from the inclusion of healthy young individuals, who had already established inner speech [10]. In fact, during reflection after the experiment, most subjects stated that they had used inner speech. In contrast, in the present study, such statements were not observed, suggesting the following possibility: some of the copying group members used outer speech in too low a voice to be heard, or they moved their lips without vocalization, with a view to promoting memorization, and this produced the same effect as outer speech. Kamoda [11] reported that the occipital lobe is bilaterally activated during visual memory tasks to reproduce memorized figures, while Sugishita indicated that the left precentral gyrus is responsible for articulation [12]. Based on these findings, it is likely that brain regions involved in the reproduction of figures vary, depending on the method of memorization, such as simply copying the figure or copying it while vocalizing or executing lip or other articulatory movements. If the subjects used articulation when copying the figure, the left precentral gyrus may have been activated with memorization, consequently producing the same effect as outer speech. This may explain the absence of significant differences in the score between the copying and outer speech groups.

During reflection upon outer speech, 7 out of the 10 outer speech group members made positive evaluations of outer speech, such as "Vocalization facilitated recall" and "The figure remained in my memory". Regarding visual stimulation, Ochi noted that memory is promoted through additional linguistic encoding [13]. Although significant differences related to this were not observed on statistical processing in the present study, outer speech was suggested to be useful for memorization and reproduction even in healthy young individuals.

The mouthpiece group showed significantly lower scores than both the copying and the outer speech groups. The presence of a mouthpiece in the mouth not only interferes with outer speech, but also prevents lip movements. As previously mentioned, lip movements facilitate memorization, and thus holding a mouthpiece in the mouth may have suppressed such movements, leading to poorer results.

On reflection after the experiment, 5 out of the 10 mouthpiece group members made negative comments, such as "The presence of the mouthpiece distracted me". The use of an unfamiliar object, such as a mouthpiece, thus may have distracted their attention, and consequently lowered their scores. It may be 
necessary to improve this method, as it may not have been appropriate to suppress outer speech in such a manner.

In short, the results of this experiment support the effectiveness of outer speech for visual memory tasks. Vocalizing the contents of such tasks may also facilitate the maintenance of memory, and enhance the effects of rehabilitation in clinical settings.

\section{Methods of Experiment 2}

\subsection{Subjects}

Thirty ( 6 males and 24 females with a mean age of $21.07 \pm 0.98$ years) students without hearing impairment, belonging to the study university, were examined with their consent. They were divided into 3 groups: copying, outer speech, and mouthpiece groups. Each group was made up of 10 members, at the same sex ratio. Furthermore, the presence of a habit of learning while watching television or listening to music was confirmed through a previous interview to allocate those who were familiar with the task at the same proportion. This study was conducted with the approval of the Medical Ethics Committee of Kanazawa University (approval number: HS27-12-1).

\subsection{Method 2}

The following noisy environment was created: the voice of a person reading out news articles was previously recorded using an IC recorder, and it was replayed during the test in a quiet, private room (length $\times$ width $\times$ height: $640 \times 620 \times$ $270 \mathrm{~cm}$ ), with a speaker placed $70 \mathrm{~cm}$ in front of the subjects (Figure 2). Using a digital noise meter, an acoustic pressure level of $60 \mathrm{~dB}$ or lower was maintained.

\subsection{Questionnaire Survey}

A questionnaire survey was conducted after the completion of the task to examine the subjects' reflection on each condition through free recall.

\subsection{Statistical Analysis}

To compare time-dependent changes in the score under each condition, a 2-way factorial analysis of variance was performed, with the condition as an unpaired

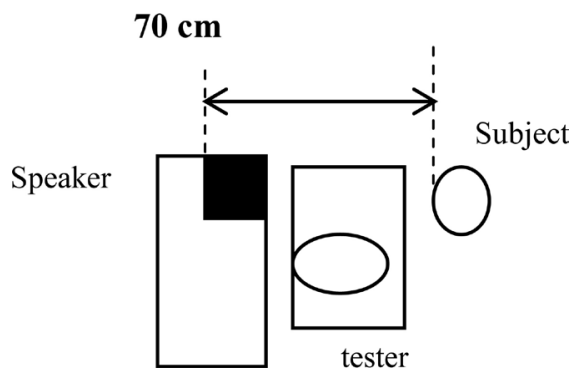

Size of the experiment room is $640 \times 620 \times 270 \mathrm{~cm}$. A tester and subject sit down to 90 degree not a meeting. Subject sits down $70 \mathrm{~cm}$ away from a speaker. From the speaker it flow news.

Figure 2. Experimental settings under the noise. 
factor and the time as a paired factor. This was followed by multiple comparisons using Tukey's method.

\section{Results of Experiment 2}

\subsection{Mean Scores under Each Condition}

Table 2 shows the mean scores and standard deviations of each group at $3 \mathrm{mi}-$ nutes and 3 days after copying.

\subsection{Time-Dependent Changes in the Score under Each Condition}

No interactions were observed under each condition. While there were no significant differences in the score between 3 minutes and 3 days after copying, it markedly varied between the copying and mouthpiece groups $(p<0.05$, Figure 3).

\subsection{Questionnaire Survey}

To the question "Did the noise weigh on your mind?", 20 and 10 out of the 30

Table 2. Mean scores and standard deviations of each group at 3 minutes and 3 days after copying (with noise).

\begin{tabular}{ccc}
\hline & \multicolumn{2}{c}{ Mean $(\mathrm{SD})$} \\
\cline { 2 - 3 } & 3 minutes later & 3 days later \\
\hline Copying group & $27.55(3.90)$ & $26.90(4.01)^{\star}$ \\
Outer speech group & $26.40(5.27)$ & $23.85(4.13)^{\star}$ \\
Mouthpiece group & $22.90(6.77)$ & $21.80(7.20)^{\star}$ \\
\hline
\end{tabular}

The full score was $36 .{ }^{*} p<0.05$.

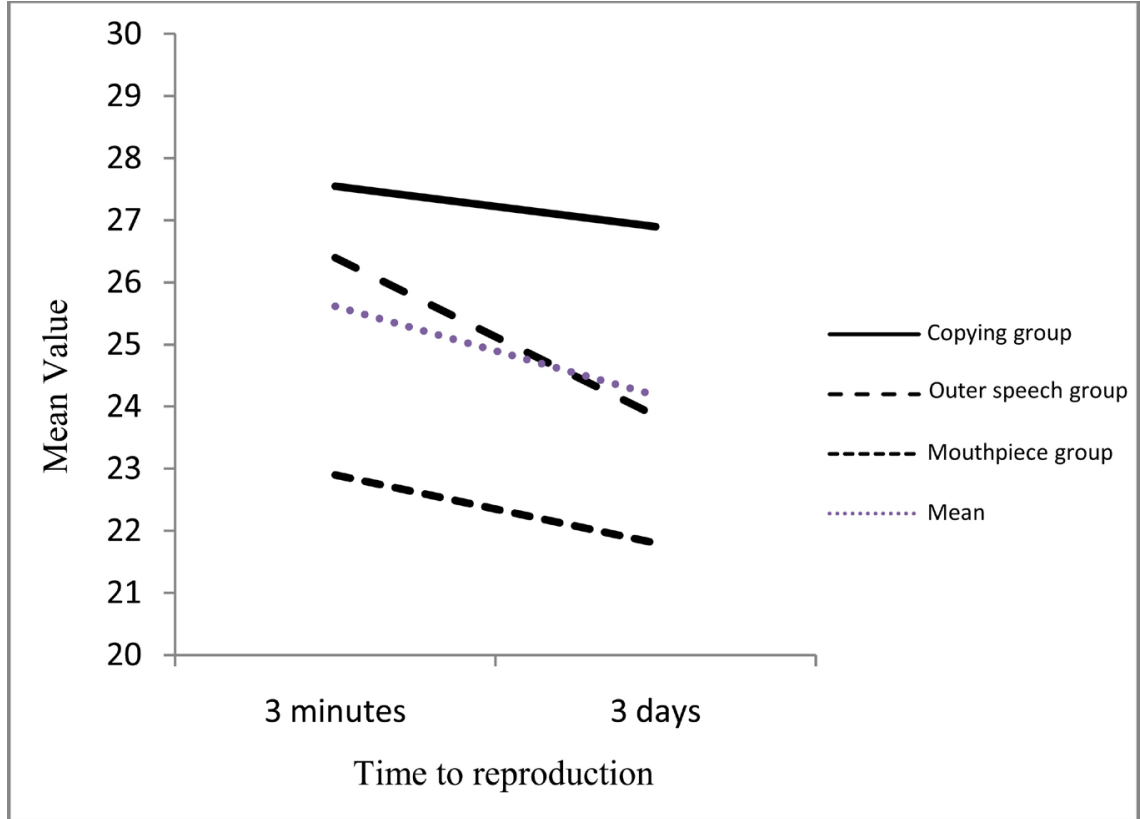

Figure 3. Time-dependent changes in the score of each group. 
subjects responded "No" and "Yes", respectively. To the question "Did the noise annoy you?", 25 and 5 responded "No" and "Yes", respectively.

To the question "What did you feel when holding a mouthpiece in your mouth?", 6 out of the 10 mouthpiece group members responded as follows: "It was difficult to concentrate" or "I had an unpleasant feeling", while 4 gave the answer "I did not feel anything".

\section{Brief Discussion}

The ROCF test was conducted, involving 30 healthy young individuals copying the figure as follows: as usual, using outer speech, or holding a mouthpiece in the mouth. The figure was reproduced and scored after 3 minutes and 3 days to examine delayed reproduction. There were no significant differences in the score between 3 minutes and 3 days after copying. On the other hand, it markedly varied between the copying and mouthpiece groups at a significance level of 0.05 . Such differences were not observed between the outer speech and mouthpiece groups.

\subsection{Comparison of the Score between 3 Minutes and 3 Days after Copying}

In this experiment with noise, there were no significant differences in the score between 3 minutes and 3 days after copying. In contrast, the score markedly varied between them in a quiet environment. In the questionnaire survey, 10 and 5 out of the 30 subjects answered that the noise weighed on their mind and annoyed them, respectively. It should be noted that the habit of learning while watching television or listening to music was present in $60 \%$ of all subjects, and their familiarity with concentrating while listening to music may have influenced their scores. For these reasons, it may be appropriate to consider that the presence of noise did not negatively affect task accomplishment in this experiment.

Takada compared learning with and without BGM, and noted a significant decrease and increase in the activities of the prefrontal and Broca areas of the brain, respectively, indicating that the memory of learned contents is maintained for a longer period in the former. It has been reported that decreases in the activity of the prefrontal area represent reduced stress, while increases in that of the Broca area reflect verbal learning and memory. If this mechanism is also applicable to the present visual memory task, the presence of noise may have reduced stress, while facilitating memory maintenance. In short, upon comparing noisy and quiet environments, the former is likely to influence subjects more positively, which may explain the favorable reproduction-related score, maintained for 3 days after copying. Another point is that a meaningful noise was used in this experiment, while Fujii et al. reported that meaningful noises were perceived as noisier than meaningless ones [14]. Based on this, meaningless noises may be more effective to achieve the above-mentioned effect, compared with meaningful noises. Further studies may be necessary to clarify appropriate types of noise for this purpose. 


\subsection{Comparison of the Score under the 3 Conditions}

The copying group showed the highest score, with significant differences from that of the mouthpiece group. Similar to the case of Experiment 1, none of the copying group members used inner speech for memorization, suggesting the possibility of some of them having used outer speech in too low a voice to be heard, or moving their lips without vocalization. Considering that lip movements are effective for memorization, as noted in Experiment 1, this may explain the copying group's score, which was markedly higher than that of the mouthpiece group.

On the other hand, the presence of a mouthpiece to suppress lip movements may have been responsible for the mouthpiece group's poor results. Furthermore, it is likely that the unpleasant feeling caused by it and reported by 6 out of the 10 members in the questionnaire survey negatively influenced their scores. Their scores were also markedly lower than those of the other groups in Experiment 1 without noise. Therefore, it was demonstrated that the suppression of lip movements with a mouthpiece affects memorization abilities, regardless of the environment.

However, there were no significant differences in the score between the outer speech and mouthpiece groups. This may be explained by the auditory masking effect. Such an effect occurs when 2 sounds are heard simultaneously, and the perception of one sound is affected by the presence of the other [15]. In Experiment 2, the subjects' voices may have been masked by the noise. The effectiveness of lip movements for memorization, confirmed through Experiment 1, may be reduced by the masking effect.

\subsection{Clinical Environments}

In a clinical setting, meaningful noises, such as surrounding people's voices, tend to be present. When performing memory tasks in such an environment, outer speech may be ineffective for memorization, as it may be masked by the noises that are present. The suppression of lip movements may also result in reduced memorization abilities.

At the same time, in noisy environments, memory may be maintained for a longer period, as suggested in the present study. However, it should be noted that the study involved healthy young individuals who were familiar with concentrating while listening to music as a factor influencing the results. In short, in the actual clinical setting involving a large number of elderly individuals, the effectiveness of memory tasks with noise is unclear, and therefore appropriate subjects should be examined.

\section{Methods of Experiment 3}

In the present study, a 3-way factorial analysis of variance was also performed, with the environment (presence/absence of noise) as the first factor (unpaired), condition (copying as usual; using outer speech; and holding a mouthpiece in the mouth) as the second factor (unpaired), and time (reproduction 3 minutes 
and 3 days after copying) as the third factor (paired).

\section{Results of Experiment 3}

The results of the 3-way factorial analysis of variance to examine interactions among the environment, condition, and time are summarized as follows: Secondary interactions were not observed, and primary interactions were limited to between the time and environment. Regarding the main effect, the values were higher when copying as usual than when holding a mouthpiece in the mouth, with $\alpha=0.05$. The simple main effect was also confirmed by lower values at 3 days than at 3 minutes after copying with or without noise, with $\alpha=0.01$.

\section{Overall Discussion}

In the present study, the ROCF test was initially conducted in a quiet environment as Experiment 1 to examine variations in the score among different methods to memorize the figure. In such an environment, there were no significant differences in the score between the copying and outer speech groups, which suggested the possibility of some of the former having used outer speech in too low a voice to be heard, or moving their lips without vocalization, achieving the same effect as outer speech, and consequently leading to the absence of differences from the outer speech group. On the other hand, the score markedly varied between the mouthpiece and copying or outer speech groups. As lip movements were suppressed in the former, the unconscious use of outer speech was also prevented, possibly leading to poor results. Based on these findings, it may be possible to enhance the effects of rehabilitation in a clinical setting by promoting patients' memorization using outer speech to vocalize the contents of training.

On examining the influences of the method to memorize the ROCF on the score in a noisy environment, it was revealed that, if memorization is performed in the presence of noise, the memory is maintained for a longer period in healthy young individuals who are familiar with concentrating while listening to music.

When memorizing the figure while holding a mouthpiece in the mouth, the score was markedly poor, regardless of the environment. On the 3-way factorial analysis of variance to examine interactions among the environment, condition, and time, the score did not vary between quiet and noisy environments, indicating the necessity of further studies to also examine appropriate volumes and types of noise.

\section{Acknowledgements}

The authors would like to thank Enago (http://www.enago.jp/) for the English language review.

\section{Conflict of Interest}

None. 


\section{References}

[1] Rivera, D., Perrin, P.B., Morlett-Paredes, A., Galarza-del-Angel, J., Martinez, C., Garza, M.T., et al. (2015) Rey-Osterrieth Complex Figure-Copy and Immediate Recall: Normative Data for the Latin American Spanish Speaking Adult Population. NeuroRehabilitaion, 37, 677-698. https://doi.org/10.3233/NRE-151285

[2] Lu Po, H., Boone, K.B., Cozolino, L. and Mirchell, C. (2003) Effectiveness of the Rey-Osterrieth Complex Figure Test and the Meyers and Meyers Recognition Trial in the Detection of Suspect Effort. Clinical Neurosciences, 17, 426-440.

[3] Silverstein, S., Osborn, L.M. and Palumbo, D.R. (1998) Rey-Osterrieth Complex Figure Test Performance in Acute, Chronic, and Remitted Schizophrenia Patients. Journal of Clinical Psychology, 54, 985-994. https://doi.org/10.1002/(SICI)1097-4679(199811)54:7<985::AID-JCLP12>3.0.CO;2$\underline{\mathrm{G}}$

[4] Shimamura, A. P., Salmon, D.P., Squire, L.R. and Butters, N. (1987) Memory Dysfunction and Word Priming in Dementia and Amnesia. Behavioral Neuroscience, 101, 347-351. https://doi.org/10.1037/0735-7044.101.3.347

[5] Cuyas, E., Verdejo-Garcia, A., Fagundo, A.B., Khymenets, O., Rodriguez, J., Cuenca, A., et al. (2011) The Influence of Genentic and Environmental Factors among MDMA Users in Cognition Performance. PLOS One, 6, e27206. https://doi.org/10.1371/journal.pone.0027206

[6] Theppitak, C., LaI, V., Izumi, H., Higuchi, Y., Kumudini, G., Movahed, M., et al. (2014) Visual Recognition Memory Test Performance was Improved in Older Adults by Extending Encoding Time and Repeating Test Trials. Journal of Occupational Health, 56, 453-460. https://doi.org/10.1539/joh.14-0021-OA

[7] Yamashita, H. (2008) Practice Effects in Repeated Administration of the Rey-Osterrieth Complex Figure: A Japanese College Students Sample Study. Neuropschology Japan, 24, 155-160.

[8] Lezak, M.D., Howieson, D.B., Bigler, E.D. and Tranel, D. (2004) Neuropsychological Assessment. Oxford University Press, Oxford, 536-541.

[9] Gallagher, C. and Burke, T. (2007) Age, Gender and IQ Effects on the Rey-Osterrieth Complex Figure Test. Journal of Clinical Psychology, 46, 35-45. https://doi.org/10.1348/014466506x106047

[10] Tsutui, M., Notoya, M., Kimura, D., Nakatani, K., Fujita, T. and Sunahara, N. (2017) Utility of Tracing as a Memory Storage Method. WJNS online

[11] Kamoda, S. (2004) A f-MRI Study on Memory Function in Normal Subjects and Patients with Partial Epilepsies. J Kanazawa Med. Univ., 29, 260-271.

[12] Sugishita, M. (2004) Aphasia Conflict. Japanese Journal of Cognitive Neuroscience, 6, 64-66.

[13] Ochi, K. (2009) Memory Promotion of Eyewitness by Memory Encoding. J Hosei Univ., 59, 63-72.

[14] Fujii, K., Yamaguchi, S., Saeki, T. (2002) Effects of Meaningful or Meaningless External Noise on Participants during Simple Mental Tasks. Humantechnology, 38, 63-68.

[15] Nanba, S. (1984) Hearing Handbook. Nakanishiya Shuppan, (Jpn), 112-138. 
Submit or recommend next manuscript to SCIRP and we will provide best service for you:

Accepting pre-submission inquiries through Email, Facebook, LinkedIn, Twitter, etc. A wide selection of journals (inclusive of 9 subjects, more than 200 journals)

Providing 24-hour high-quality service

User-friendly online submission system

Fair and swift peer-review system

Efficient typesetting and proofreading procedure

Display of the result of downloads and visits, as well as the number of cited articles Maximum dissemination of your research work

Submit your manuscript at: http://papersubmission.scirp.org/

Or contact wins@scirp.org 